\title{
Coherent Structural Dynamics of a Prototypical Charge-Density-Wave-to-Metal Transition
}

\author{
T. Huber, ${ }^{1,}$ S. O. Mariager, ${ }^{2}$ A. Ferrer, ${ }^{1,2}$ H. Schäfer, ${ }^{3}$ J. A. Johnson, ${ }^{2}$ S. Grübel, ${ }^{2}$ A. Lübcke, ${ }^{2,5}$ L. Huber, ${ }^{1}$ T. Kubacka, ${ }^{1}$ \\ C. Dornes, ${ }^{1}$ C. Laulhe, ${ }^{6,7}$ S. Ravy, ${ }^{6}$ G. Ingold, ${ }^{2}$ P. Beaud, ${ }^{2}$ J. Demsar, ${ }^{3,4}$ and S. L. Johnson ${ }^{1}$ \\ ${ }^{1}$ Institute for Quantum Electronics, Physics Department, ETH Zurich, CH-8093 Zurich, Switzerland \\ ${ }^{2}$ Swiss Light Source, Paul Scherrer Institut, CH-5232 Villigen PSI, Switzerland \\ ${ }^{3}$ Physics Department, Universität Konstanz, D-78457 Konstanz, Germany \\ ${ }^{4}$ Institute of Physics, Ilmenau University of Technology, D-98693 Ilmenau, Germany \\ ${ }^{5}$ Laboratoire de Spectroscopie Ultrarapide, EPF Lausanne, CH-1015 Lausanne, Switzerland \\ ${ }^{6}$ Synchrotron SOLEIL, L'Orme des Merisiers, Saint-Aubin, BP 48, FR-91192 Gif-sur-Yvette Cedex, France \\ ${ }^{7}$ Université Paris-Sud, 91405 Orsay Cedex, France \\ (Received 29 April 2014; published 11 July 2014)
}

\begin{abstract}
Using femtosecond time-resolved x-ray diffraction, we directly monitor the coherent lattice dynamics through an ultrafast charge-density-wave-to-metal transition in the prototypical Peierls system $\mathrm{K}_{0.3} \mathrm{MoO}_{3}$ over a wide range of relevant excitation fluences. While in the low fluence regime we directly follow the structural dynamics associated with the collective amplitude mode; for fluences above the melting threshold of the electronic density modulation we observe a transient recovery of the periodic lattice distortion. We can describe these structural dynamics as a motion along the coordinate of the Peierls distortion triggered by the prompt collapse of electronic order after photoexcitation. The results indicate that the dynamics of a structural symmetry-breaking transition are determined by a high-symmetry excited state potential energy surface distinct from that of the initial low-temperature state.
\end{abstract}

DOI: 10.1103/PhysRevLett.113.026401

PACS numbers: 71.45.Lr, 73.20.Mf, 78.47.J-

The notion of an ultrafast or nonthermal phase transition has become a widely used concept in nonequilibrium solid state physics. Usually it refers to the transition to a transient state of different symmetry after intense photoexcitation with a femtosecond laser pulse. A prime example of such a transition is the destruction of electronic order in a charge density wave (CDW) compound, and ultrafast transitions from numerous broken symmetry states in correlated electron systems have been observed [1-5].

ACDW system presents a unique model system since only two coupled subsystems with long-range order existelectronic order and the lattice - and both are accessible with modern time-resolved techniques. The dynamics and the melting of the electronic order in CDW systems have been studied in great detail $[4,6,7]$, but mostly with methods that are only indirectly sensitive to the structure of the solid. Optical pump-probe spectroscopy can in principle look at structural symmetry changes by investigating the disappearance of coherent oscillations from zone-folded modes [4,5]. These structural contributions to the optical properties are, however, often difficult to distinguish from purely electronic contributions, particularly when the relevant vibrational modes become strongly damped.

A direct view of the dynamics of the lattice can be obtained by methods directly sensitive to the positions of lattice atoms in solids. Time resolved x-ray diffraction $[2,8]$ and electron diffraction methods $[3,9,10]$ are emerging, but the reported measurements so far lack either sufficient time resolution or sensitivity to track possible coherent structural dynamics associated with the ultrafast melting of electronic order. This information is crucial to fully understand an ultrafast symmetry breaking transition, and to answer questions concerning the time scale of the symmetry change and the nature of the nonequilibrium state that exists on a picosecond time scale after excitation.

In this Letter we show that by following the coherent structural dynamics through an ultrafast photoinduced CDW-to-metal transition with sufficient time resolution over a wide range of relevant excitation fluences, we can see the time scale of the structural symmetry change and the dynamics of the periodic lattice distortion (PLD) in the transient high symmetry state.

As shown by Peierls, a one-dimensional conductor is fundamentally unstable with respect to the formation of a broken symmetry ground state [11], the CDW ground state. Below the metal-to-CDW transition temperature $T_{c}$, the equilibrium electronic density and lattice positions are modulated with a wave vector $\mathbf{q}_{\mathrm{CDW}}$ that is related to the Fermi surface nesting condition of the system. In thermodynamic equilibrium, a multicomponent complex order parameter combines electronic and structural order and fully characterizes the broken symmetry state [12]. A periodic lattice distortion is associated with CDW formation. New collective excitations of the complex order parameter arise, leading to Raman active phonon modes that show softening toward $T_{c}$.

A prototypical Peierls transition is realized in the quasione-dimensional conductor $\mathrm{K}_{0.3} \mathrm{MoO}_{3}$ (often termed Blue 
Bronze) [13-15]. $\mathrm{K}_{0.3} \mathrm{MoO}_{3}$ is widely regarded as a textbook example of Fermi surface nesting driven CDW formation, displaying a clear correlation between $\mathbf{q}_{\mathrm{CDW}}$ and the Fermi nesting vector $[16,17]$ as well as a Kohn anomaly above $T_{c}$ [18]. Upon cooling below $T_{c}=183 \mathrm{~K}$, $\mathrm{K}_{0.3} \mathrm{MoO}_{3}$ undergoes a second order phase transition and develops an incommensurate CDW [19-21] with wave vector $\mathbf{q}_{\mathrm{CDW}}=\left(\begin{array}{lll}1 & q_{b} & 0.5\end{array}\right)$. The modulation along the $\mathbf{b}^{*}$ axis varies with temperature [22]; at $100 \mathrm{~K} q_{b}=0.748(1)$ [23]. The lowest frequency Raman active collective excitation at $\nu_{\mathrm{AM}}=1.7 \mathrm{THz}$ [18] is commonly referred to as the amplitude mode (AM). At room temperature, $\mathrm{K}_{0.3} \mathrm{MoO}_{3}$ has space group $C 2 / m$ and can be cleaved along the $(20 \overline{1})$ surface, in which linear chains of cornerand edge-sharing $\mathrm{MoO}_{6}$ octahedra are located [see Fig. 1(a)]. The chains are separated by layers of K-atoms. Static grazing incidence $\mathrm{x}$-ray diffraction data show that the CDW modulation vector is the same in the surface layers as in the bulk [24].

The time-resolved $\mathrm{x}$-ray diffraction measurements were carried out at a hard $x$-ray synchroton slicing source [25] in a grazing incidence geometry [see Fig. 1(b)]. The cleaved $\mathrm{K}_{0.3} \mathrm{MoO}_{3}$ single crystal sample was cooled well below $T_{c}$ to $T_{0}=95 \mathrm{~K}$ using a cryogenic nitrogen blower. The crystal

(a)

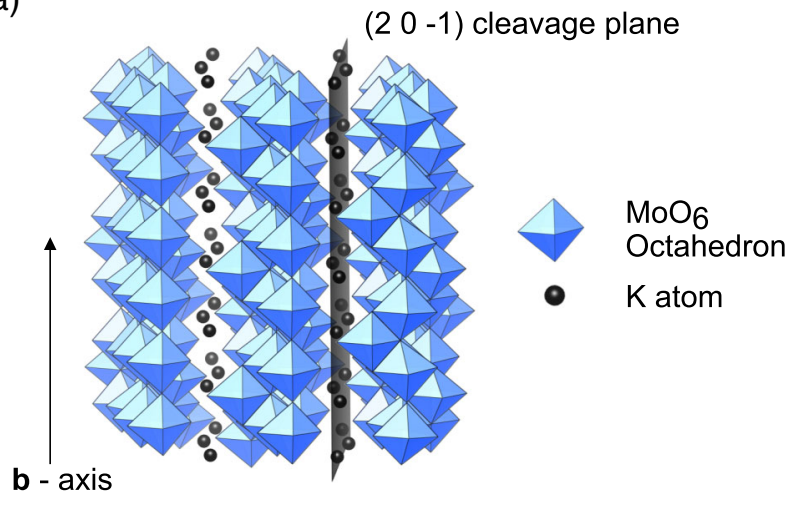

(b)

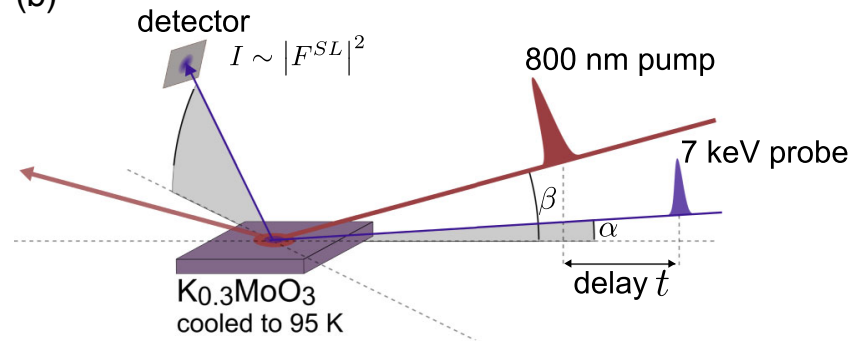

FIG. 1 (color online). (a) Linear chains of high conductivity composed of $\mathrm{MoO}_{6}$ octahedra, separated by layers of K-atoms. The plane corresponds to the (201) cleavage plane. (b) Experimental setup; parameters as described in the text. The incidence plane of $\mathrm{x}$-ray probe and $800 \mathrm{~nm}$ pump beam is vertical. The detector is positioned to measure the intensity of a SL reflection, providing a direct view of the structural symmetry of the system. was excited by $1.55 \mathrm{eV}(800 \mathrm{~nm})$ laser pulses with a duration (full width at half maximum) of $100 \mathrm{fs}$, hitting the sample at a grazing angle of $\beta=10^{\circ}$ with $p$ polarization. The penetration depth of the excitation pulses is around $\delta_{L}=80 \mathrm{~nm}$ [26]. The pulse length of the sliced $\mathrm{x}$-ray probe pulses is estimated to be $t_{\text {probe }}=80 \mathrm{fs}$. The x-ray energy was set to $7 \mathrm{keV}$, resulting in a penetration depth of around $\delta_{X}=100 \mathrm{~nm}$ for a grazing angle $\alpha \approx 0.4^{\circ}$. The $\mathrm{x}$-ray beam was focused to $10 \mu \mathrm{m}$ vertically and $300 \mu \mathrm{m}$ horizontally, ensuring a homogeneous lateral excitation of the probed volume.

The intensity of the $\left(1\left(4-q_{b}\right) \overline{0.5}\right)$ superlattice (SL) reflection was measured with an avalanche photodiode for varying time delays between the pump and the x-ray probe. Due to the low scattering efficiency of the SL reflection, count rates of only 2 photons/s at the detector could be achieved, thus requiring integration times of up to 12 hours for a single trace with sufficient signal-to-noise ratio. To prevent $\mathrm{x}$-ray induced sample degradation, the $\mathrm{K}_{0.3} \mathrm{MoO}_{3}$ sample was never exposed to the full power of the unsliced synchrotron radiation.

For excitation fluences below the melting threshold of the CDW condensate, we can directly follow the structural dynamics associated with the collective AM in a system exhibiting a Peierls transition. Figure 2 shows the time evolution of the measured SL diffraction intensity $I(t)$, normalized to the equilibrium diffraction intensity $I_{0}=I(t<0)$. The small diffraction efficiency of the SL reflection allows us to apply the kinematic approximation $I \propto\left|F^{\mathrm{SL}}\right|^{2}$, where $F^{\mathrm{SL}}$ is the structure factor for the measured SL reflection. Since the amplitude of the equilibrium PLD is small [23], to leading order we estimate $F^{\mathrm{SL}}$ as $F^{\mathrm{SL}}(t) \propto X(t)$, where $X(t)$ describes the atomic motion along the structural coordinate of the Peierls distortion. Since the AM corresponds to the atomic movement $X(t)$ along the distortion coordinate, we model it in the

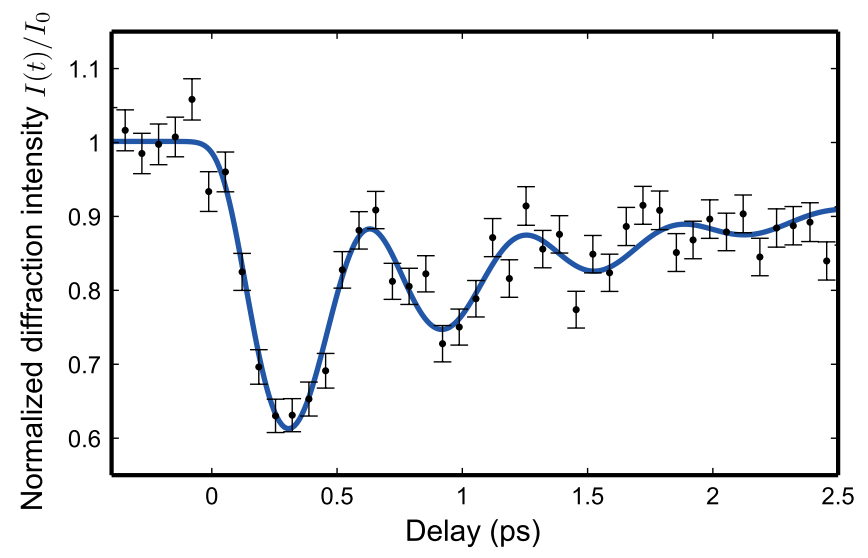

FIG. 2 (color online). Time evolution of the normalized peak diffraction intensity of the $\left(1\left(4-q_{b}\right) \overline{0.5}\right)$ reflection for a pump fluence $F=0.3 \mathrm{~mJ} / \mathrm{cm}^{2}$, well below the melting threshold of the CDW condensate. The error bars correspond to photon counting statistics. The solid line is a fit to the data using a displacive excitation model [see Eq. (1)]. 
displacive limit of a stimulated Raman scattering process $[27,28]$. The displacive excitation model yields [29]

$$
x(t)=A_{\text {disp }}\left(\cos \left(2 \pi \nu_{\mathrm{AM}} t\right) e^{-t / \tau_{\mathrm{AM}}}-e^{-t / \tau_{\text {disp }}}\right)+1,
$$

where the structural coordinate $x(t)=X(t) / X_{T}$ is normalized to the equilibrium distortion $X_{T}$. The first term in Eq. (1) corresponds to the damped coherent oscillation of the AM with frequency $\nu_{\mathrm{AM}}$ and damping time $\tau_{\mathrm{AM}}$, while the second term describes the relaxation of the transient quasiequilibrium position after excitation with a time constant $\tau_{\text {disp. }}$. In Fig. 2 we show a fit of the normalized diffraction intensity $I(t) / I_{0}=|x(t)|^{2}$ according to the model to the measured diffraction intensity. For an excitation fluence [30] $F=0.3 \mathrm{~mJ} / \mathrm{cm}^{2}$, the values obtained from the fit are $\nu_{\mathrm{AM}}=1.62 \pm 0.1 \mathrm{THz}, \tau_{\mathrm{AM}}=0.6 \pm 0.15 \mathrm{ps}$ and $\tau_{\text {disp }}=2.3 \pm 0.4 \mathrm{ps}$. The values for $\nu_{\mathrm{AM}}$ and $\tau_{\mathrm{AM}}$ are in close agreement with results obtained from optical measurements at $100 \mathrm{~K}$ [6].

In Fig. 3(a) we present the time evolution of the SL peak diffraction intensity for a range of excitation fluences, providing a complete picture of the coherent lattice dynamics through the CDW-to-metal transition. The amplitude of the coherent oscillation associated with the AM first increases with increasing fluence, but at a fluence $F=$ $1 \mathrm{~mJ} / \mathrm{cm}^{2}$ no clear signature of the AM can be observed. The relaxation time scale of the SL peak intensity increases to $\tau_{\text {disp }} \approx 10$ ps and the measured time scale of the initial drop of SL diffraction intensity reduces to $\tau_{\mathrm{drop}} \approx 100 \mathrm{fs}$, the experimental time resolution. The SL diffraction intensity right after the initial drop for $F=1 \mathrm{~mJ} / \mathrm{cm}^{2}$ reduces to approximately $0.5 I_{0}$ and remains at this value for higher excitation fluences. Similarly, the drop in SL diffraction intensity at a time delay $t=3 \mathrm{ps}$ remains at approximately $0.4 I_{0}$ and does not recover within the measured time window for fluences above $F=1 \mathrm{~mJ} / \mathrm{cm}^{2}$. We attribute the background level to contributions from unexcited volumes of the sample resulting from surface steps of the cleaved crystal [31]. The fluences for which the saturation background level is reached agree well with the fluences for which in optical pump-probe spectroscopy coherent oscillations associated with zone-folded modes were observed to disappear [4].

For fluences well above this threshold, we observe a transient recovery of the SL intensity, as demonstrated by the data recorded at $F=2.1 \mathrm{~mJ} / \mathrm{cm}^{2}$ and $F=3.7 \mathrm{~mJ} / \mathrm{cm}^{2}$. After the initial suppression, the SL diffraction intensity recovers and peaks at $t=350 \mathrm{fs}$. Note that when taking into account the finite time resolution of the experiment, the recovered SL intensity at this time corresponds to nearly 80 $\%$ of the equilibrium SL diffraction intensity.

To describe the coherent dynamics of the PLD in the transient regime after photoexcitation $(t>0)$, we employ a simple phenomenological model. Assuming that the CDW in $\mathrm{K}_{0.3} \mathrm{MoO}_{3}$ is driven by Fermi surface nesting, we introduce a fluence dependent double-well potential $[32,33]$,

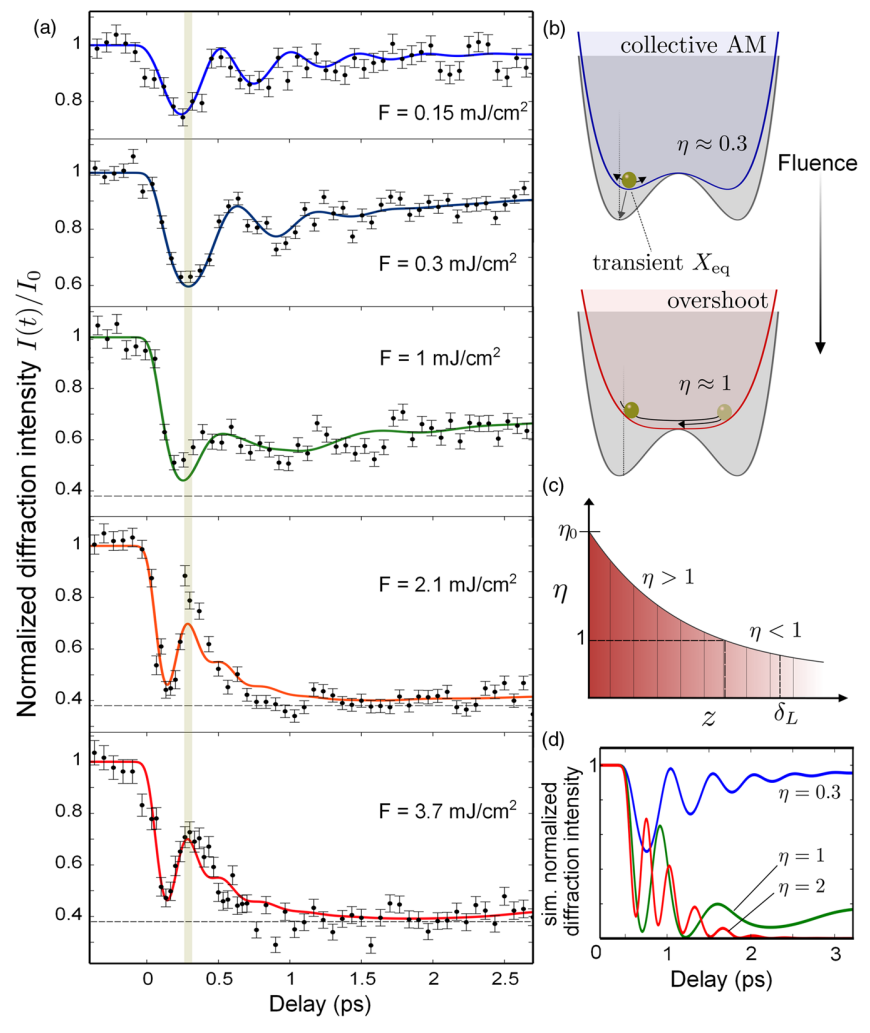

FIG. 3 (color online). (a) Time evolution of the normalized SL diffraction peak intensity for increasing fluence $F$. The horizontal dashed line in the higher fluence scan represents the saturation background. Solid lines are fits according to the model as described in the text. (b) Photoinduced changes of the doublewell potential as assumed in our model simulation. The grey potential corresponds to the unperturbed CDW state (where the excitation parameter $\eta=0$ ), the green circle represents the displacement along the structural coordinate of the Peierls distortion. The transient quasiequilibrium position after excitation is labeled $X_{\text {eq }}$. (c) Schematic of the inhomogeneously excited crystal in the case of a high fluence excitation that leads to $\eta_{0}>1$, the $z$ axis corresponds to the axis perpendicular to the surface plane. (d) Simulated diffraction signal of a thin layer for certain values of $\eta$ (at $t=0$ ), where $\delta_{L}$ corresponds to the laser penetration depth.

$$
V(x)=\frac{1}{2}\left(\eta \exp \left(-\frac{t}{\tau_{\text {disp }}}\right)-1\right) x^{2}+\frac{1}{4} x^{4}
$$

Here, $\eta \exp \left(-t / \tau_{\text {disp }}\right)$ describes the transient change in the potential energy caused by laser excitation. The parameter $\eta \propto F$ is related to the suppression of electronic order just after excitation, while $\tau_{\text {disp }}$ specifies the time scale of relaxation back to the initial potential. In an unperturbed system $\eta=0$ and the potential is partitioned by a Peierls barrier. The system resides in one of the minimum positions, corresponding to the equilibrium displacement along the structural coordinate of the Peierls distortion [see Fig. 3(b)]. The coherent structural motion along this coordinate after photoexcitation can then be determined by the following equation of motion: 


$$
\begin{aligned}
& \frac{1}{\omega_{\mathrm{DW}}^{2}} \frac{\partial^{2}}{\partial t^{2}} x-\left(1-\eta \exp \left(-\frac{t}{\tau_{\mathrm{disp}}}\right)\right) x+x^{3} \\
& +\frac{2 \gamma(t)}{\omega_{\mathrm{DW}}^{2}} \frac{\partial}{\partial t} x=0,
\end{aligned}
$$

where $\omega_{\text {DW }}$ corresponds to the angular frequency of the motion in the double-well (DW) potential. In the equation of motion, we introduce a phenomenological damping parameter, which we will discuss later. In the low perturbation regime $(\eta \approx 0)$ corresponding to small amplitudes of the structural motion and a small reduction of the barrier height, the results of the harmonic displacive model [Eq. (1)] are reproduced. For fluences above the melting threshold of the CDW condensate, $\eta \geq 1$ and the CDW condensate is depleted, causing a nearly instantaneous collapse of the Peierls barrier. Subsequently, the structure evolves in a high-symmetry potential and overshoots into the opposite side of the potential that was before partitioned by the barrier, corresponding to a transient PLD with the opposite phase.

To finally compare the experimental data to the model simulations, we must take into account the inhomogeneous excitation profile of the single crystal sample. The measured signal contains contributions from layers with different excitation levels. We calculate the integrated intensity $I(t)$ measured by the detector by incoherently summing the contributions from a series of layers at various distances $z$ from the surface, each with a thickness of $d=10 \mathrm{~nm}$ [34]: $I(t) \propto \sum_{j}^{\infty} \Delta_{j}\left|F_{j}^{\mathrm{SL}}(t)\right|^{2}$. Here, $F_{j}^{\mathrm{SL}}(t)$ corresponds to the structure factor of the $j$ th layer [see Fig. 3(c)], and we weight its contribution to the measured signal with $\Delta_{j}=$ $\exp \left(-2 j d / \delta_{X}\right)$ to account for $\mathrm{x}$-ray absorption in the crystal. To fit the data, we solve the equation of motion [Eq. (3)] for each layer. The simulated signal resulting from only one layer is depicted in Fig. 3(d) for selected values of $\eta$ at $t=0$. The only fluence dependent parameters needed to fit the whole data set are $\eta_{0}$, the value of $\eta$ in the uppermost surface layer for $t=0$, and the relaxation time $\tau_{\text {disp }}$ of the Peierls barrier averaged over all layers. The frequency $\nu_{\mathrm{DW}}=\omega_{\mathrm{DW}} / 2 \pi$ in the equation of motion for the double-well potential is determined by fits to the low fluence traces to be $\nu_{\mathrm{DW}}=1.53 \mathrm{THz}$, in close agreement with the high temperature bare phonon frequency of the phonon branch displaying the Kohn anomaly [18].

In the transient regime after intense photoexcitation, the damping parameter $\gamma(t)$ can have several possible physical origins. Electron-phonon coupling and lattice anharmonicities should both contribute to the damping of the motion along the distortion coordinate, but the system is far away from equilibrium and possible microscopic scattering channels are not easily elucidated. With a constant damping parameter, we cannot account for the near full transient recovery of the PLD, followed by a complete suppression after only one cycle. We thus introduce a phenomenological time-dependent damping term $\gamma(t)=$ $\gamma_{\text {asym }}\left(1-e^{-t / \tau_{\gamma}}\right)^{2}$, where $\tau_{\gamma}=300 \mathrm{fs}$, close to the fast relaxation time scale measured with optical pump-probe spectroscopy [4]. We chose an expression as simple as possible, while still capturing the main features of the data. As an asymptotic value we use $\gamma_{\text {asym }}=2 \mathrm{ps}^{-1}$, which is close to the damping constant measured via neutron diffraction near the thermal phase transition [18]. The time scale $\tau_{\gamma}$ could be related to electronic relaxation processes after photoexcitation $[6,33]$. However, the unusual time dependent damping could also be caused by anharmonic coupling in the transient regime, where eigenmodes are not well defined and strong time- dependent coupling effects might arise [7]. To further investigate the scattering mechanisms after intense photoexcitation and to specify the damping mechanism in the transient regime, emerging time-resolved diffuse scattering techniques will be useful [35].

The model simulations agree well with the measured data [see Fig. 3(a)], reproducing all critical details of the evolution through all fluence regimes: (i) excitation of collective AM for low fluences $\rightarrow$ (ii) no clear signature of AM and increased suppression of the PLD $\rightarrow$ (iii) transient recovery of PLD, followed by a complete suppression of the PLD. In the intermediate fluence regime $\left(F=1 \mathrm{~mJ} / \mathrm{cm}^{2}\right)$, the agreement between the simulation and the data is not as good. This may indicate that our estimate of the excitation depth profile as a simple exponential is oversimplified, leading to an error in the relative magnitude of contributions from highly excited regions near the surface and less excited regions further into the bulk of the crystal. We have not considered in the model any possible nonlinear components to the initial absorption process or subsequent effects from hot electron diffusion, both of which could modify the shape of the excitation profile. For excitation levels far above or far below the melting threshold, the data and simulation are both less sensitive to the detailed excitation profile since the dynamics of all excited layers are similar.

It is important to note that within the framework of this model, we rely on the assumption that the complete structural dynamics associated with the CDW-to-metal transition can be described as a coherent motion along a single degree of freedom, namely the motion along the coordinate of the Peierls distortion. Domain growth and the creation of an incoherent phonon population leading to a change in the Debye-Waller factor do not need to be included in the phenomenological description to capture all important features of the data [32].

The agreement with the model indicates that during the ultrafast melting of a CDW, the structural dynamics are determined by the properties of the high-symmetry phase and not by the lattice modes of the initial state. This has wide implications for the understanding of materials with a predominantly electronically driven PLD that is the result of a coupling of the lattice to long-range electronic order. Ultimately, it explains why the dynamics of structural symmetry during a so-called nonthermal phase transition can be fast, while time scales observed during an adiabatic soft-mode phase transition diverge when approaching $T_{c}$. 
In summary, we observed the coherent lattice dynamics associated with CDW formation in a Peierls system. The prototypical nature of the quasi-one-dimensional conductor $\mathrm{K}_{0.3} \mathrm{MoO}_{3}$ allows us to interpret the results as a structural motion along one generalized coordinate in an excitation dependent double-well potential, triggered by the collapse of electronic order. To explain the nearly full transient recovery of the PLD following the initial complete suppression, we introduce a phenomenological time-dependent damping factor. The physical origin of this time-dependent damping is unclear, but may indicate strongly timedependent electron-phonon or phonon-phonon coupling. It will be very illustrative to test if similar overshoot dynamics can be observed in systems where the full mechanism of the transition is not as clear-cut [36], e.g., systems displaying a two-dimensional CDW [10,37].

Time resolved $\mathrm{x}$-ray diffraction measurements were carried out at the X05LA beam line, and preparative static grazing incidence diffraction measurements were performed at the X04SA beam line of the Swiss Light Source, Paul Scherrer Institut, Villigen. We thank R. Merlin for helpful discussions and P. Willmott, D. Grolimund, and C. Borca for experimental help. We acknowledge financial support by the NCCR Molecular Ultrafast Science and Technology (NCCR MUST), a research instrument of the Swiss National Science Foundation (SNSF).

*tihuber@phys.ethz.ch

[1] F. Schmitt et al., Science 321, 1649 (2008).

[2] P. Beaud, S. Johnson, E. Vorobeva, U. Staub, R. Souza, C. Milne, Q. Jia, and G. Ingold, Phys. Rev. Lett. 103, 155702 (2009).

[3] M. Eichberger, H. Schäfer, M. Krumova, M. Beyer, J. Demsar, H. Berger, G. Moriena, G. Sciaini, and R. J. D. Miller, Nature (London) 468, 799 (2010).

[4] A. Tomeljak, H. Schäfer, D. Städter, M. Beyer, K. Biljakovic, and J. Demsar, Phys. Rev. Lett. 102, 066404 (2009).

[5] S. Wall, D. Wegkamp, L. Foglia, K. Appavoo, J. Nag, R. F. Haglund, J. Stähler, and M. Wolf, Nat. Commun. 3, 721 (2012).

[6] H. Schäfer, V. V. Kabanov, M. Beyer, K. Biljakovic, and J. Demsar, Phys. Rev. Lett. 105, 066402 (2010).

[7] H. Y. Liu et al., Phys. Rev. B 88, 045104 (2013).

[8] E. Möhr-Vorobeva, S. L. Johnson, P. Beaud, U. Staub, R. De Souza, C. Milne, G. Ingold, J. Demsar, H. Schaefer, and A. Titov, Phys. Rev. Lett. 107, 036403 (2011).

[9] G. Sciaini, M. Harb, S. G. Kruglik, T. Payer, C. T. Hebeisen, F.-J. Meyer zu Heringdorf, M. Yamaguchi, M. Horn-von Hoegen, R. Ernstorfer, and R. J. D. Miller, Nature (London) 458, 56 (2009).

[10] T.-R. T. Han, Z. Tao, S. D. Mahanti, K. Chang, C.-Y. Ruan, C. D. Malliakas, and M. G. Kanatzidis, Phys. Rev. B 86, 075145 (2012).

[11] R. E. Peierls, Quantum Theory of Solids (Oxford University Press, New York, 1955).
[12] G. Grüner, Density Waves in Solids (Addison-Wesley, Reading, MA, 1994).

[13] W. Fogle and J.H. Perlstein, Phys. Rev. B 6, 1402 (1972).

[14] J. P. Pouget, S. Kagoshima, C. Schlenker, and J. Marcus, J. Phys. (Paris), Lett. 44, 113 (1983).

[15] G. Travaglini, P. Wachter, Solid State Commun. 37, 599 (1981).

[16] G. H. Gweon et al., J. Phys. Condens. Matter 8, 9923 (1996).

[17] A. V. Fedorov, S. A. Brazovskii, V. N. Muthukumar, P. D. Johnson, J. Xue, L.-C. Duda, K. E. Smith, W. H. McCarroll, M. Greenblatt, and S. L. Hulbert, J. Phys. Condens. Matter 12, L191 (2000).

[18] J. P. Pouget, B. Hennion, C. Escribe-Filippini, and M. Sato, Phys. Rev. B 43, 8421 (1991).

[19] I. M. G. Travaglini and P. Wachter, Solid State Commun. 45, 289 (1983).

[20] J. P. Pouget, C. Noguera, A. H. Moudden, and R. Moret, J. Phys. (Paris) 46, 1731 (1985).

[21] P. A. Lee, T. M. Rice, and P. W. Anderson, Phys. Rev. Lett. 31, 462 (1973).

[22] R. M. Fleming, L. F. Schneemeyer, and D. E. Moncton, Phys. Rev. B 31, 899 (1985).

[23] W. J. Schutte and J. L. De Boer, Acta Crystallogr. Sect. B 49, 579 (1993).

[24] X. M. Zhu, R. Moret, H. Zabel, I. Robinson, E. Vlieg, and R. Fleming, Phys. Rev. B 42, 8791 (1990).

[25] P. Beaud, S. Johnson, A. Streun, R. Abela, D. Abramsohn, D. Grolimund, F. Krasniqi, T. Schmidt, V. Schlott, and G. Ingold, Phys. Rev. Lett. 99, 174801 (2007).

[26] Calculated with optical data from Ref. [19].

[27] H. J. Zeiger, J. Vidal, T. Cheng, E. Ippen, G. Dresselhaus, and M. Dresselhaus, Phys. Rev. B 45, 768 (1992).

[28] T. E. Stevens, J. Kuhl, and R. Merlin, Phys. Rev. B 65, 144304 (2002).

[29] As in [27] when $1 / \tau_{\text {disp }}, 1 / \tau_{\mathrm{AM}}<2 \pi \nu_{\mathrm{AM}}$.

[30] All fluences in this Letter correspond to absorbed fluences, calculated with optical conductivity data published in [19].

[31] Using white light interferometry, step heights up to $1 \mu \mathrm{m}$ were measured on the sample surface, which is much bigger than the laser penetration depth $\delta_{L}$. At varying grazing incidence angles $\alpha$ below the angle of total reflection a constant SL diffraction signal could be measured, which we attribute to diffraction from surface irregularities.

[32] R. Yusupov, T. Mertelj, V.V. Kabanov, S. Brazovskii, P. Kusar, J.-H. Chu, I. R. Fisher, and D. Mihailovic, Nat. Phys. 6, 681 (2010).

[33] H. Schaefer, V. V. Kabanov, and J. Demsar, Phys. Rev. B 89, 045106 (2014).

[34] The approximation of the signal as an incoherent sum of the contributions of the layers is appropriate for a grazing incidence geometry with shallow beam exit angles and a detector larger than the spot size of the diffracted beam. These conditions are met for the SL reflection under study.

[35] M. Trigo et al., Nat. Phys. 9, 790 (2013).

[36] M. D. Johannes and I. I. Mazin, Phys. Rev. B 77, 165135 (2008).

[37] J. Demsar, L. Forro, H. Berger, and D. Mihailovic, Phys. Rev. B 66, 041101 (2002). 